\title{
Histograms for Fuzzy Color Spaces
}

\author{
J. Chamorro-Martínez, D. Sánchez, J.M. Soto-Hidalgo, P. Martínez-Jiménez
}

\begin{abstract}
In this paper we introduce two kinds of fuzzy histograms on the basis of fuzzy colors in a fuzzy color space and the notion of gradual number by Dubois and Prade. Fuzzy color spaces are a collection of fuzzy sets providing a suitable, conceptual quantization with soft boundaries of crisp color spaces. Gradual numbers assign numbers to values of a relevance scale, typically $[0,1]$. Contrary to convex fuzzy subsets of numbers (called fuzzy numbers, but corresponding to fuzzy intervals as an assignment of intervals to values of $[0,1])$, they provide a more precise representation of the cardinality of a fuzzy set. Histograms based on gradual numbers are particularly well-suited for serving as input to another process. On the contrary, they are not the best choice when showing the information to a human user. For this second case, linguistic labels represented by fuzzy numbers are a better alternative, so we define linguistic histograms as an assignment of linguistic labels to each fuzzy color. We provide a way to calculate linguistic histograms based on the compatibility between gradual numbers and linguistic labels. We illustrate our proposals with some examples.
\end{abstract}

J. Chamorro-Martínez, D. Sánchez, P. Martínez-Jiménez

Dept. Computer Science and A.I., University of Granada, Spain.

e-mail: \{jesus,daniel,pedromartinez $\} @$ decsai.ugr.es

D. Sánchez

European Centre for Soft Computing, Mieres, Spain. e-mail: daniel.sanchezf@ softcomputing.es

J.M. Soto-Hidalgo

Department of Computer Architecture, Electronics and Electronic Technology, University of Córdoba, Spain. e-mail: jmsoto@uco.es

This work has been supported by the Spanish Science and Innovation Ministry under the project TIN2009-08296. 


\section{Introduction}

Histograms are the basis of many techniques for image restoration, enhancement, segmentation, retrieval, etc. In principle, a color histogram is defined as a function $h\left(\mathbf{c}_{\mathbf{k}}\right)=n_{k}$ where $\mathbf{c}_{\mathbf{k}}=[x, y, z]$ is a color and $n_{k}$ is the number of pixels in the image having the color $\mathbf{c}_{\mathbf{k}}$. It is common to normalize a histogram by dividing each of its values by the total number of pixels, obtaining the frequency of occurrence of a color $\mathbf{c}_{\mathbf{k}}$.

This simple approach has the drawback that a color space is not representative of the collection of colors we can distinguish and identify. In addition, the values $n_{k}$ use to be very low because there are many colors in a crisp color space, and it is easy to find small color variations in real images. Since in practice many of the colors $\mathbf{c}_{\mathbf{k}}$ are indistinguishable for us, a solution is to use an histogram defined on groups of indistinguishable colors $C_{k}$, in which the associated number of pixels is $h\left(C_{k}\right)=\sum_{\mathbf{c}_{\mathbf{k}} \in C_{K}} n_{k}$. The collection of groups of colors $C_{1} \ldots C_{n}$ defines a partition (quantization) of the color space employed.

Many approaches for defining the quantization of the color space are available in the literature. In this work we are interested in fuzzy approaches [10, 12, 14, $21,24,19,20]$, in which each group of colors is in fact a fuzzy subset of colors, called a fuzzy color, and the whole collection of colors defining a fuzzy partition of the color space is called a fuzzy color space. This approach has the advantage that it is able to represent the fact that indistinguishability is a fuzzy, gradual concept for we humans, i.e. for us, colors are indistinguishable to a certain degree. Crisp boundaries inherent to crisp quantization are counterintuitive for us. In addition, both the (fuzzy) quantization and the histograms are less sensitive to small variations of the boundaries

In the literature there are several proposals which define histograms over a set of fuzzy colors $[4,9,15,16]$. One drawback of most of these proposals is that they work only with intensities. In addition, the counting of fuzzy colors is performed by using the sigma-count (i.e., the sum of membership degrees). However, the sigmacount is not a suitable measure of cardinality in many applications, as it has been recognized by several authors [8]. Proposals based on the sigma-count summarize the counting into a single number, so they do not represent the fuzziness of the count itself.

In [1], we introduced an approach to fuzzy histograms based on the concept of fuzzy natural number introduced in [3]. This approach represented a kind of compromise between precision and understandability. In this work we introduce two approaches, a numerical one intended to provide a more accurate representation of cardinalities on the basis of gradual numbers, and a linguistic one obtained from the previous one, intended for improving understandability at the cost of precision.

The rest of the paper is organized as follows: in section 2 we recall the notion of fuzzy color space. Different approaches to fuzzy cardinality and their suitability for histogram representation on fuzzy color spaces are discussed in section 3 . We introduce our new histograms and some examples in section 4. Finally, the main conclusions and future works are summarized in section 5 . 


\section{Fuzzy color spaces}

In order to represent the semantic compatibility between crisp colors and linguistic color terms, in [20] we introduce the following definitions of fuzzy color and fuzzy color space on a generic crisp color space $X Y Z$ with domain of components being $D_{X}, D_{Y}$, and $D_{Z}$ :

Definition 1. [20] A fuzzy color $\widetilde{C}$ is a linguistic label whose semantics is represented in a color space $X Y Z$ by a normalized fuzzy subset of $D_{X} \times D_{Y} \times D_{Z}$.

Definition 2. [20] A fuzzy color space $\widetilde{X Y Z}$ is a set of fuzzy colors $\widetilde{C}_{1}, \ldots, \widetilde{C}_{m}$ that define a fuzzy partition of $D_{X} \times D_{Y} \times D_{Z}$, i.e., that satisfies:

1. $\bigcup_{\{1, \ldots, m\}} \sup \left(\widetilde{C}_{i}\right)=X Y Z$, i.e., the union of the support of the $\widetilde{C}_{i}$ covers the whole space.

2. $\operatorname{ker}\left(\widetilde{C}_{i}\right) \cap \operatorname{ker}\left(\widetilde{C}_{j}\right)=\emptyset \forall i \neq j$, i.e., the kernels of the $\widetilde{C}_{i}$ and $\widetilde{C}_{j}$ are pairwise disjoint.

3. $\forall i \in\{1, \ldots, m\} \exists \mathbf{c} \in X Y Z$ such that $\widetilde{C}_{i}(\mathbf{c})=1$, i.e., there is at least one object fully representative of the fuzzy color $\widetilde{C}_{i}$.

Condition 3 is always verified by definition of fuzzy color. Condition 1 implies $\forall \mathbf{c} \in X Y Z \exists i \in\{1, \ldots, m\}$ such that $\widetilde{C}_{i}(\mathbf{c})>0$. Conditions 2 and 3 imply $\widetilde{C}_{i} \not \subset \widetilde{C}_{j}$ $\forall i \neq j$.

In [20] we proposed several fuzzy color spaces using color names provided by the well-known ISCC-NBS system [11]. ISCC-NBS provides several color sets in the form of sets of pairs (linguistic term, crisp color). Using the methodology introduced in [20], we calculate for each color set a fuzzy color space on the basis of a Voronoi diagram of the crisp color space, calculated using the crisp colors of the set of pairs considered. The Voronoi diagram is a crisp partition corresponding to the 0.5 -cut of the fuzzy colors. The kernel and support of each fuzzy color is obtained as a scaling with parameters $\alpha$ and $\beta$ respectively, with $\alpha<1<\beta$, and guaranteeing the conditions in definition 2. The membership functions of the fuzzy colors are obtained on the basis of distances in the crisp color space. For more details see [20].

In [20], we have obtained three fuzzy color spaces on the basis of the sets of color names Basic (13 colors), Extended (31 colors) and Complete (267 colors) in the RGB color space. For instance, the Basic set has color names corresponding to ten basic color terms (pink, red, orange, yellow, brown, olive, green, blue, violet, purple), and 3 achromatic ones (white, gray, and black). The corresponding representative crisp colors are shown in figure 1, together with a rough view of the core, the alpha-cuts of level 0.5, and the support of some fuzzy colors in the fuzzy color space obtained from ISCC-NBS Basic in [20]. We are not showing examples of the fuzzy color spaces for the sets Extended and Complete because of the lack of space. 


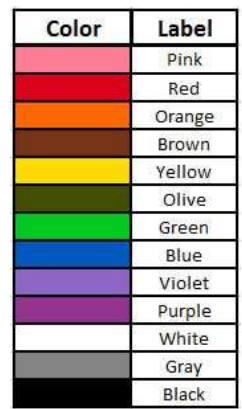

(a)

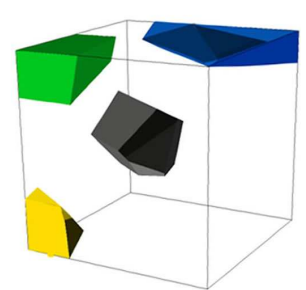

(d)

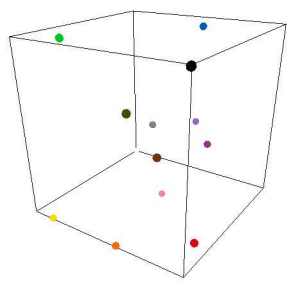

(b)

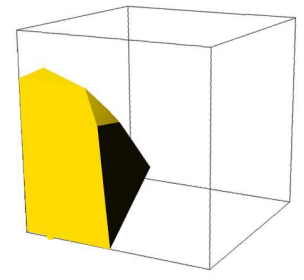

(e)

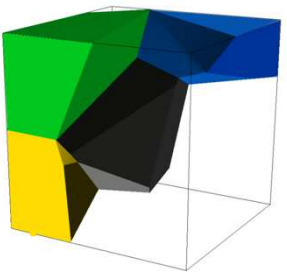

(c)

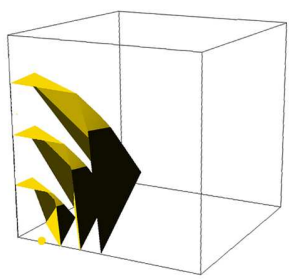

(f)

Fig. 1 Part of the RGB fuzzy color space obtained in [20] from the ISCC-NBS Basic set of colors. (a) ISCC-NBS Basic set of colors (representative crisp color and color name). (b) Situation of the representative crisp colors in the RGB color space. (c) Volumes of colors in the 0.5-cut for the fuzzy colors yellow, blue, green, and gray obtained from the Voronoi diagram in the RGB cube. (d) Volumes of colors in the kernel of the same fuzzy colors. (e) Volume of colors in the support of the fuzzy color yellow. (f) Superimposed views of part of the surfaces of the volumes of colors in the kernel (most internal), 0.5-cut (middle) and support (most external) for the fuzzy color yellow.

\section{Cardinality of fuzzy sets}

In order to define histograms on the basis of fuzzy color spaces, we need to cope with the problem of cardinality of fuzzy sets, since the set of pixels painted in a certain fuzzy color is in fact a fuzzy set whose cardinality must appear in the histogram. In this section we recall the most employed approaches for solving the problem of cardinality of fuzzy sets, and we discuss on their advantages and disadvantages, particularly in the definition of histograms. 


\subsection{Scalar cardinalities}

In these approaches, the cardinality of a fuzzy set is a crisp number, either real or integer [22]. The most employed cardinality for fuzzy sets is the scalar sigma-count, defined for any fuzzy set $F: X \rightarrow[0,1]$ as

$$
s c(F)=\sum_{x \in X} F(x)
$$

However, the sigma-count is not a suitable measure of cardinality in many applications. In fact, sc was introduced as a measure of energy of a fuzzy set [13]. Sigma-count is counterintuitive since $s c$ is not a natural number in general. Even if the result is a natural number, we can obtain the same result in very different situations, thus loosing information. This is the case if we consider two situations like having 100 pixels compatible with a color $\widetilde{C}$ to a degree 0.1 , and having 10 pixels compatible with $\widetilde{C}$ to a degree 1 ; in both cases, the result of the sigma count applied to the fuzzy set of pixels compatible with $\widetilde{C}$ is 10 . Some alternatives consider in the addition only values above a certain threshold, but this does not solve other problems, as we shall see.

\subsection{Fuzzy numbers}

The next approach in popularity is to consider that the cardinality of a fuzzy set is a fuzzy number, i.e., a normalized, convex subset of the real line or the nonnegative integers [5, 22]. However, in [2] we showed that in some cases this is counterintuitive. Consider for example the fuzzy set given by $A=1 / x_{1}+0.5 / x_{2}+0.5 / x_{3}$. The cardinality of $A$ could be one (because $x_{1}$ belongs to $A$ for sure) or, if we relax our criterion to accept elements in $A$, the cardinality could be three (accepting $x_{2}$ and $x_{3}$ belong to $A$ as well). However, the cardinality cannot be two, since if $x_{2} \in A$ then $x_{3} \in A$ and vice versa. This way, the cardinality is not convex. In addition, this example illustrates that the sigma-count is not always a good measure since $s c(A)=2$.

Several authors have related this issue to the idea that the possible cardinalities of a fuzzy set are the cardinalities of its $\alpha$-cuts, since these are the possible crisp representatives of the fuzzy set, and hence that should be the support of the fuzzy cardinality [2]. In our previous example the possible cardinalities of $A$ are 1 or 3 since its possible $\alpha$-cuts are $\left\{x_{1}\right\}$ and $\left\{x_{1}, x_{2}, x_{3}\right\}$. Several alternative proposals that comply with this idea are available $[23,2]$. In [1] we introduced histograms on fuzzy color spaces in which the cardinality associated to each fuzzy color was a fuzzy subset of the naturals calculated by using method $E D$, proposed in [2]. This fuzzy set is not convex in general. We will see an example later. 


\subsection{Gradual numbers}

In [7], Dubois and Prade introduced the ideas of gradual element and gradual number as a way to represent fuzzy quantities. Gradual numbers assign numbers to values of a relevance scale, typically $[0,1]$. The cardinality of a fuzzy set can be represented by a gradual number in which the cardinality of the $\alpha$-cut of the fuzzy set is assigned to $\alpha$.

Following the notation in [17], a gradual (real) number is a pair $(\Lambda, \mathscr{R})$ where $\Lambda \subset(0,1]$ is finite, and $\mathscr{R}:(0,1] \rightarrow \mathbb{R}$. Let $\mathbb{R}_{R L}$ be the set of gradual real numbers. Operations are extended as follows:

Definition 3. Let $f: \mathbb{R}^{n} \rightarrow \mathbb{R}$ and let $R_{1} \ldots R_{n}$ be gradual numbers. Then $f\left(R_{1}, \ldots, R_{n}\right)$ is a gradual number with

$$
\Lambda_{f\left(R_{1}, \ldots, R_{n}\right)}=\bigcup_{1 \leq i \leq n} \Lambda_{R_{i}}
$$

and, $\forall \alpha \in \Lambda_{f\left(R_{1}, \ldots, R_{n}\right)}$

$$
\mathscr{R}_{f\left(R_{1}, \ldots, R_{n}\right)}(\alpha)=f\left(\mathscr{R}_{R_{1}}(\alpha), \ldots, \mathscr{R}_{R_{n}}(\alpha)\right)
$$

Gradual numbers offer several advantages. First, they don't introduce imprecision in the cardinality, since each $\alpha$-cut is assigned a crisp number. On the contrary, the $\alpha$-cut of a fuzzy number is an interval where the cardinality is assumed to be, and hence it is an imprecise representation of the cardinality. Hence, the so-called fuzzy numbers are in fact fuzzy intervals [6]. Another advantage with respect to fuzzy numbers is that gradual numbers have the same algebraic structure as ordinary numbers, whist fuzzy numbers satisfy the properties of interval arithmetic only. In addition, gradual numbers do not increase imprecision of the representation with operations, even to the extent that operations on gradual numbers may yield crisp numbers. In particular, it is always the case that for any gradual number $a$, $a-a=0, a / a=1$ (provided $a \neq 0$ ), etc. They are hence a precise and very useful representation when the cardinality is to be employed in any calculation.

However, gradual numbers are not as intuitive as fuzzy numbers when the cardinality is to be expressed to a human user. In particular, it is difficult to provide a linguistic label describing precisely a gradual number. Another disadvantage is related to the space needed for representing a gradual number, and the time needed for arithmetic calculations, both being proportional to the number of $\alpha$-cuts employed. In practice, only a finite number of cuts is necessary, corresponding to levels in the representation of the gradual number, see [17].

\subsection{Fuzzy cardinality and histograms on fuzzy color spaces}

Summarizing, we conclude the following: 
- Scalar cardinalities are not accurate representations of the cardinality. They can be seen as summaries of the real cardinality, either by discarding the cardinality of all but one of the $\alpha$-cuts, or by providing the center of gravity, like in the case of the sigma-count (this interpretation is given in [2]).

- Fuzzy numbers represent restrictions, linguistic concepts on numbers, but are not well suited for providing accurate representations, since the latter are non-convex in general [2]. Their arithmetics is an arithmetic of restrictions (fuzzy intervals), that is not appropriate for representing cardinalities that are to be employed in further calculations. On the contrary, they are the best choice for summarizing the cardinality into a linguistic term to be provided to human users (losing information and accuracy, but less than scalar cardinalities).

- Gradual numbers are the most accurate representation of cardinality, and the best choice for representing cardinalities to be used in further calculations. They are not the best choice for giving results to the user.

In [1] we employed non-convex fuzzy subsets of the non-negative integers for defining fuzzy histograms. This approach represents a compromise between the advantages and disadvantages of using fuzzy numbers and gradual numbers. In this paper, we propose to define two kinds of histograms: one based on gradual numbers, to be used in practice by the computer for image processing, and an approximation of this histogram by means of linguistic labels (fuzzy numbers) in order to give information to the user. These are introduced and illustrated in the next section.

\section{New Histograms on Fuzzy Color Spaces}

\subsection{Gradual histogram}

Definition 4. A gradual color histogram is a function $h_{G}$ that assigns a gradual number to every fuzzy color in a fuzzy color space $\widetilde{X Y Z}$.

The gradual number corresponding to every fuzzy color $\widetilde{C}$ is obtained by assigning to $\alpha$ the cardinality of the $\alpha$-cut of the fuzzy subset of pixels with color $\widetilde{C}$. Since the fuzzy subset of pixels with color $\widetilde{C}$ is finite, in practice we need only a finite number of cuts for representing the gradual number. However, this number may be large, so in some cases it may be interesting to consider just a fixed collection of cuts with equidistant values of $\alpha$. See [17] for further discussion on that.

Let us remark that the gradual integer number can be easily transformed into a gradual rational number by dividing the number associated to each level $\alpha$ by the total number of pixels in the image. This is convenient when we want the histogram to represent proportions instead of absolute values. We shall see examples in section 4.3.

Finally, usual image processing operations performed on crisp histograms can be extended directly to gradual histograms by using definition 3 , i.e., by applying the operation in each level. 


\subsection{Linguistic histogram}

Let $\widetilde{X Y Z}$ be a fuzzy color space. Let us consider a collection of fuzzy linguistic quantifiers $S_{Q}=\left\{Q_{1} \ldots Q_{k}\right\}$ provided by the user, like Around $10 \%$ or Most, represented by appropriate fuzzy subsets of the unit interval, and Between 50 and 100, represented by appropriate fuzzy subsets of the non-negative integers.

Definition 5. A linguistic color histogram is a function $h_{L}$ that assigns a linguistic quantifier from $S_{Q}$ to every fuzzy color in $\widehat{X Y Z}$.

We consider user-defined quantifiers in order to improve understandability, since accuracy of the linguistic approach is always worst than that of the gradual approach.

In order to obtain the quantifier for every fuzzy color $\widetilde{C}$, we take the gradual number associated to $\widetilde{C}$ in the gradual color histogram (an accurate representation of cardinality) and we calculate the compatibility between this gradual number and every quantifier in $S_{Q}$. The quantifier that yields maximum compatibility is then chosen to represent the linguistic amount or proportion of pixels having color in the linguistic histogram. The compatibility is calculated by evaluating the accomplishment degree of the quantified statement $Q_{i}$ of pixels in the image are painted in $\widetilde{C}$, by using the method introduced in [18] as follows: let $(\Lambda, \rho)$ be the gradual number representing the cardinality of the fuzzy subset of pixels with color $\widetilde{C}$. Let $\Lambda=\left\{\alpha_{1}, \ldots, \alpha_{r}\right\}$ with $1=\alpha_{1}>\alpha_{2}>\cdots>\alpha_{r}>\alpha_{r+1}=0$, with $r>0$. Then the evaluation of $Q_{i}$ of pixels in the image are painted in $\widetilde{C}$ is a number in $[0,1]$ given by equation 4

$$
\sum_{\alpha_{i} \in \Lambda}\left(\alpha_{i}-\alpha_{i+1}\right) \times Q\left(\rho\left(\alpha_{i}\right)\right)
$$

\subsection{A first synthetic example}

Our first example, taken from [17], allows us to illustrate the differences between the different approaches to cardinality of fuzzy sets discussed in section 3. The fuzzy color space employed here, described in [17], is not that of section 2, but this is unimportant for our purpose in this first example. Figure 2 shows two images, the first one containing eight colors in the kernel of different fuzzy colors. Let $\widetilde{C_{1}}, \ldots, \widetilde{C_{8}}$ denote these colors, from left to right and top to bottom (see [17] for a definition of the membership functions). In the second one we have four colors that are compatible to a degree 0.5 with two of the eight fuzzy colors in the first image.

Table 1 shows the (relative) cardinality of the fuzzy set of pixels painted in each fuzzy color in both images, using different approaches. The result is the same for all the fuzzy colors in both images by the way they have been defined. In the case of image A, since the eight crisp colors employed are in the kernel of fuzzy colors, the sets of pixels painted in every fuzzy color are crisp, and hence a crisp cardinality is obtained. In particular, the fuzzy number for image A is the same despite 


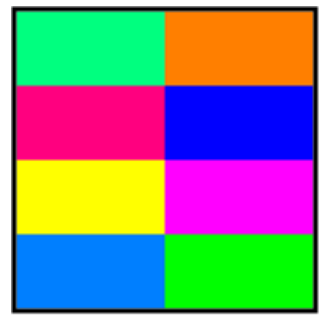

A

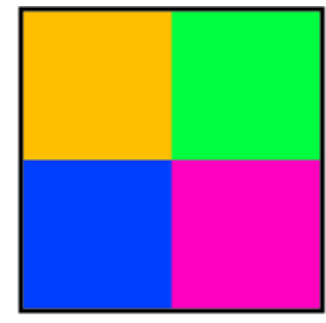

B

Fig. 2 Two synthetic images.

the method employed for calculating it. In the gradual approach, crisp results are represented by the fact that only the level $\alpha=1$ is necessary, since all the levels are assigned the same cardinality (in this case, 1/8). In the case of the quantifier, any quantifier having $1 / 8$ in its core will give a result of 1 in the evaluation of the quantified sentence. We assume here that the user has predefined a collection of quantifiers of the form Around $x \%$, with $x \in[0,1]$ corresponding to some kind of fuzzy partition of the unit interval. Under this assumption, Around $12.5 \%$ is a triangular fuzzy number/quantifier with kernel $1 / 8$ with appropriate specificity for the user. Of course, in this particular case, it is clear that a quantifier Exactly $12.5 \%$ would have been a better choice.

\begin{tabular}{|l||l||l|}
\hline Quantifier & Around 12.5\% & Around 0\% or Around 25\% \\
\hline \hline Gradual & $1 \mapsto 1 / 8$ & $\begin{array}{l}1 \mapsto 0 \\
\end{array}$ \\
& $0.5 \mapsto 1 / 4$ \\
\hline \hline Fuzzy number & $1 /(1 / 8)$ & $1 / 0+\sum_{0<x \leq 1 / 4} 0.5 / x$ \\
\hline \hline Scalar & $1 / 8$ & $1 / 8$ \\
\hline \hline & Image A & Image B \\
\hline
\end{tabular}

Table 1 Cardinalities (same for all fuzzy colors $\widetilde{C_{1}}, \ldots, \widetilde{C_{8}}$ ) in the Color Histograms obtained for images $\mathrm{A}$ and $\mathrm{B}$ in figure 2 by using different approaches to cardinality.

In the case of image B, the first thing to highlight is the fact that the scalar cardinality yields the same result than that of image A. However, the perception of the colors that appear in the image and the corresponding frequencies is very different in both images, the only possible frequencies being intuitively $1 / 8$ in image $\mathrm{A}$, and 0 and $1 / 4$ in image B. This example illustrates how the sigma-count is not an accurate measure in general. Notice also that using a threshold below $1 / 2$ does not solve the problem.

The fuzzy cardinality provided is the one that would be provided by most existing methods, and represents the notion of approximately Between 0 and 1/4. Though a suitable information for a human user, it is not an accurate representation, since there is no chance that the cardinality is other than 0 or $1 / 4$. However, notice that it yields a different result than that for image $\mathrm{A}$, and hence it is more accurate than the 
sigma-count. As a final remark, the fuzzy cardinality defined in [2] and employed in [17] gives a fuzzy set $0.5 / 0+0.5 /(1 / 4)$ which is not a fuzzy number since it is neither normalized nor convex. This is more accurate than a fuzzy number and, though good enough to be easily understandable by a human, this is not always the case, as the fuzzy set can have a large support. Hence, it is a kind of compromise between accuracy and understandability.

The gradual approach gives also a different result for both images, in this case representing accurately the only possible cardinalities in both images, associated to different levels. In addition, this representation is very easy to use for further calculations, since we have a crisp number in each level, and any kind of calculation to be employed is performed independently in each level following definition 3 . The results obtained in each level can later be summarized using techniques similar to those employed here to provide a quantifier from a gradual number.

Finally, the evaluation of quantified sentences would provide for image B a fulfilment degree of 0.5 for both triangular quantifiers Around 0\% and Around 25\%. This is in consonance with our intuition about the possible cardinalities in image B. Here, two different options are to choose one of them on the basis of other application or user-specific information (for example, whether we prefer to be conservative or not with respect to the amount of colors), or to give both of them. Notice that this case is rather unfrequent, and it is motivated by the fact that very specific, synthetic images with particular memberships have been employed.

\subsection{A real example}

Let us consider the real image in figure 3. We are not giving fuzzy and gradual results here because of lack of space, being also little informative in a real case to the user. We have considered a collection of ten triangular relative quantifiers defining a fuzzy partition in Ruspini's sense, with kernels being the percentages $0,1,2,3$, $4,12,25,50,75$, and 100 . Let us denote by $Q P_{x}$ the quantifier with kernel $x$. Table 2 shows the (approximate) value obtained by evaluation of the quantified sentences $Q P_{x}$ of the pixels are painted in color $\widetilde{C}$ for every fuzzy color $\widetilde{C}$ in the fuzzy color space associated to the ISCC-NBS Basic set of colors explained in section 2. The last column contains the values of the linguistic histogram.

Let us remark that the addition of the result of the evaluation of quantified sentences is not necessarily 1 , since these are not frequencies but values of compatibility between cardinality and quantifiers. Similarly, the addition of the quantities indicated in the linguistic histogram is not expected to be $100 \%$ in general, since these are fuzzy sets around the value. Specificity also play a role here, for example, the collection of quantifiers employed here "jumps" from $4 \%$ to $12 \%$, hence the latter quantifier is much less specific than those between 0-4\%. In practice, we are just working on the basis of the quantifiers the user is interested in, his/her vocabulary, and the results may vary depending on the number and definition of quantifiers, and the fuzzy color space employed. However, we think that the results are compati- 


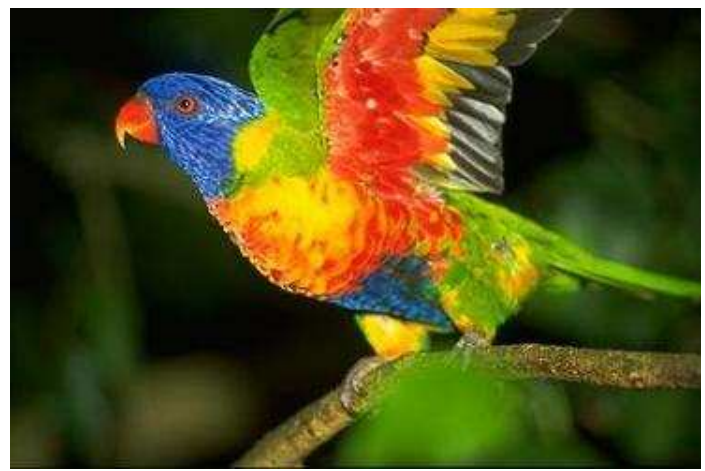

Fig. 3 Parrot image.

\begin{tabular}{|l||l|l|l|l|l|l|l|l|l|l||c|}
\hline \multicolumn{1}{|c|}{ Fuzzy Color } & \multicolumn{9}{|c||}{ Quantifiers } & Linguistic Histogram \\
\cline { 2 - 8 } \multicolumn{1}{c||}{$\widetilde{C}$} & $Q P_{0}$ & $Q P_{1}$ & $Q P_{2}$ & $Q P_{3}$ & $Q P_{4}$ & $Q P_{12}$ & $Q P_{25}$ & $Q P_{50}$ & $Q P_{75}$ & $Q P_{100}$ & $h_{L}(\widetilde{C})$ \\
\hline Pink & $\mathbf{0 . 4 7}$ & 0.29 & 0.14 & 0.08 & 0 & 0 & 0 & 0 & 0 & 0 & Around 0\% \\
\hline Red & 0.20 & $\mathbf{0 . 2 5}$ & 0.16 & 0.15 & 0.21 & 0 & 0 & 0 & 0 & 0 & Around 1\% \\
\hline Orange & 0 & 0.03 & 0.11 & 0.06 & 0.31 & $\mathbf{0 . 4 3}$ & 0.03 & 0 & 0 & 0 & Around 12\% \\
\hline Brown & 0.12 & 0.17 & 0.10 & 0.09 & 0.22 & $\mathbf{0 . 2 5}$ & 0.01 & 0 & 0 & 0 & Around 12\% \\
\hline Yellow & 0 & 0 & 0.13 & 0.14 & $\mathbf{0 . 3 9}$ & 0.32 & 0 & 0 & 0 & 0 & Around 12\% \\
\hline Olive & 0 & 0 & 0 & 0 & 0.02 & 0.19 & 0.35 & $\mathbf{0 . 4 1}$ & 0.01 & 0 & Around 50\% \\
\hline Green & 0.10 & 0.12 & 0.13 & 0.15 & $\mathbf{0 . 1 6}$ & 0.13 & 0.05 & 0 & 0 & 0 & Around 4\% \\
\hline Blue & 0.25 & $\mathbf{0 . 2 6}$ & 0.22 & 0.23 & 0.02 & 0 & 0 & 0 & 0 & 0 & Around 1\% \\
\hline Violet & $\mathbf{0 . 4 7}$ & 0.42 & 0.09 & 0 & 0 & 0 & 0 & 0 & 0 & 0 & Around 0\% \\
\hline Purple & $\mathbf{0 . 7 3}$ & 0.16 & 0.08 & 0.02 & 0 & 0 & 0 & 0 & 0 & 0 & Around 0\% \\
\hline White & $\mathbf{0 . 8 5}$ & 0.08 & 0.03 & 0 & 0 & 0 & 0 & 0 & 0 & 0 & Around 0\% \\
\hline Gray & 0.03 & 0.21 & 0.13 & 0.10 & 0.18 & $\mathbf{0 . 2 6}$ & 0.05 & 0 & 0 & 0 & Around 12\% \\
\hline Black & 0 & 0 & 0 & 0 & 0 & 0.06 & $\mathbf{0 . 6 2}$ & 0.31 & 0 & 0 & Around 25\% \\
\hline
\end{tabular}

Table 2 Evaluation of quantified sentences and linguistic color histogram for figure 3.

ble with what we can see in the image. Finally, choosing more than one quantifier when the difference in the accomplishment degree is very low can be an interesting alternative in some cases, like in the case of the fuzzy color Green.

\section{Conclusions and future work}

Different color histograms of images, based on different approaches to the cardinality of fuzzy sets, may be applied depending on the use they are intended. We propose to use gradual histograms when the result is to be employed as the basis for further calculation in the setting of image processing and analysis, whilst we consider linguistic histograms more appropriate when the information is to be given to the user. As future work we plan to use these histograms in developing applications for linguistic description of images and image information retrieval. 


\section{References}

1. J. Chamorro-Martínez, D. Sánchez, and J.M. Soto-Hidalgo. A novel histogram definition for fuzzy color spaces. In Proceedings IEEE WCCI 2008, pages 2149 - 2156, 2008.

2. M. Delgado, M.J. Martín-Bautista, D. Sánchez, and M.A. Vila. A probabilistic definition of a nonconvex fuzzy cardinality. Fuzzy Sets and Systems, 126(2):41-54, 2002.

3. M. Delgado, M.J. Martín-Bautista, D. Sánchez, and M.A. Vila. Fuzzy integers: Representation and arithmetic. In Proceedings of IFSA'05, 2005.

4. A. Doulamis and N. Doulamis. Fuzzy histograms for efficient visual content representation: application to content-based image retrieval. In IEEE International Conference on Multimedia and Expo, pages 893-896, Aug 2001.

5. D. Dubois and H. Prade. Fuzzy cardinality and the modeling of imprecise quantification. Fuzzy Sets and Systems, 16:199-230, 1985.

6. D. Dubois and H. Prade. Fuzzy intervals versus fuzzy numbers: Is there a missing concept in fuzzy set theory? In Linz Seminar 2005 Abstracts, pages 45-46. 2005.

7. D. Dubois and H. Prade. Gradual elements in a fuzzy set. Soft Computing, 12:165-175, 2008.

8. D. Dubois, H. Prade, and T. Sudkamp. A discussion of indices for the evaluation of fuzzy associations in relational databases. In T. Bilgic, B. De Baets, and O. Kaynak, editors, Fuzzy Sets and Systems - Proceedings IFSA'03, LNAI 2715, pages 111-118. Springer, 2003.

9. J. Han and Kai-Kuang. Fuzzy color histogram and its use in color image retrieval. IEEE Transactions on Image Processing, 11(8):944-952, 2002.

10. L. Hildebrand and M. Fathi. Knowledge-based fuzzy color processing. IEEE. Tran. on Systems, Man and Cybernetics. Part C, 34(4):499-505, November 2004.

11. K.L. Kelly and D.B. Judd. Color: universal language and dictionary of names. National Bureau of Standards (USA), (440), 1976.

12. G. Louverdis, I. Andreadis, and Ph. Tsalides. New fuzzy model for morphological colour image processing. In IEEE Proc. Vis. Image Signal Proc., volume 149, pages 129-139, 2002.

13. A. De Luca and S. Termini. A definition of a nonprobabilistic entropy in the setting of fuzzy sets theory. Information and Control, 20:301-312, 1972.

14. T. Mitsuishi, N. Kayaki, and K. Saigusa. Color construction using dual fuzzy system. In IEEE Int. Sym. Comp. Intelligence for Measurement Sys. and Appl., pages 136-139, 2003.

15. S. Romani, P. Sobrebilla, and E. Montseny. Obtaining the relevant colors of an image through stability-based fuzzy color histograms. In IEEE International Conference on Fuzzy Systems, volume 2, pages 914-919. St. Louis, Missouri (USA), May 2003.

16. T.A. Runkler. Fuzzy histograms and fuzzy chi-squared tests for independence. In IEEE International Conference on Fuzzy Systems, volume 3, pages 1361-1366, July 2004.

17. D. Sánchez, M. Delgado, and M.A. Vila. RL-numbers: An alternative to fuzzy numbers for the representation of imprecise quantities. In Proc. Fuzz-IEEE 2008, pages 2058-2065, 2008.

18. D. Sánchez, M. Delgado, and M.A. Vila. Fuzzy quantification using restriction levels. In Proc. WILF 2009., volume 5571 of LNCS, pages 28-35. Springer, 2009.

19. M. Seaborn, L. Hepplewhite, and J. Stonham. Fuzzy colour category map for the measurement of colour similarity and dissimilarity. Pattern Recognition, 38(4):165-177, 2005.

20. J.M. Soto-Hidalgo, J. Chamorro-Martínez, and D. Sánchez. A new approach for defining a fuzzy color space. In Proceedings IEEE WCCI 2010, pages 292-297, 2010.

21. N. Sugano. Color-naming system using fuzzy set theoretical approach. In IEEE Int. Conf. on Fuzzy Systems, pages 81-84, 2001.

22. M. Wygralak. Cardinalities of Fuzzy Sets. Springer, 2003.

23. L. A. Zadeh. A theory of approximate reasoning. Machine Intelligence, 9:149-194, 1979.

24. H. Zhu, H. Zhang, and Y. Yu. Deep into color names: Matching color descriptions by their fuzzy semantics. LNAI 4183, pages 138-149, 2006. 